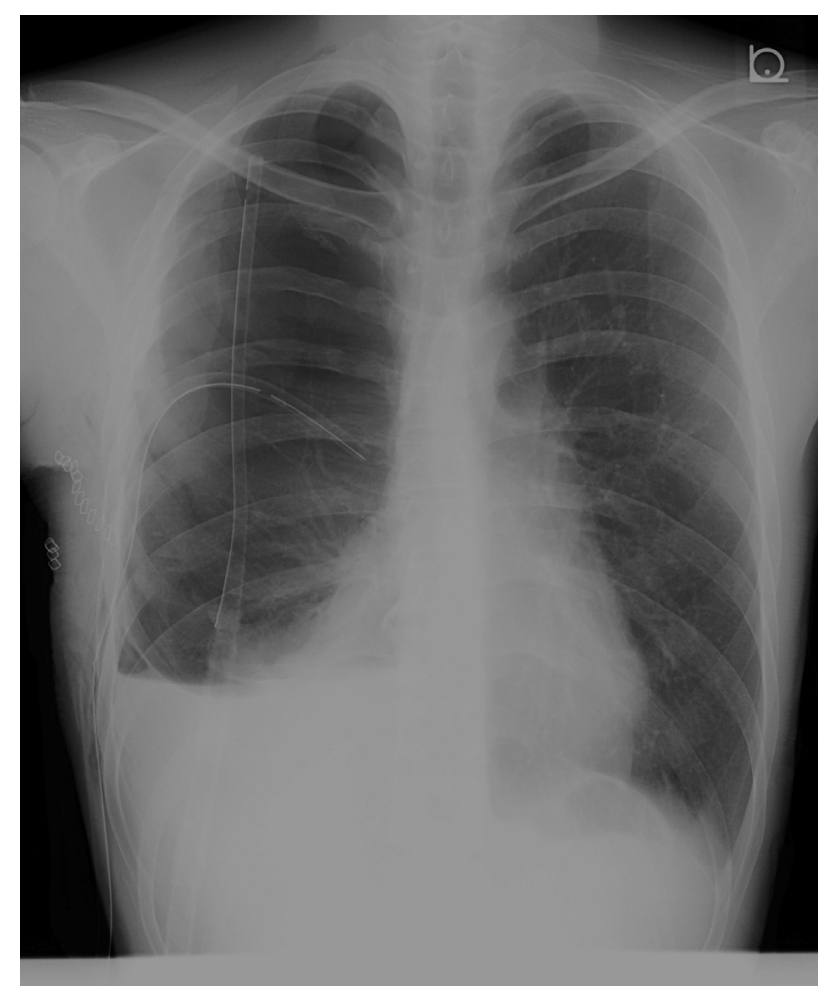

FIGURE 2. Chest radiograph demonstrating that after the additional drainage with suction, the lung became partially reinflated. Note the abnormal vasculature of the hilum.

malpositioning of the lung at the end of thoracic surgery. ${ }^{1,2}$ It is considered that complete deflation of the lung with a double-lumen tube makes torsion possible. ${ }^{3,4}$ Other reported causes of lobar torsion include trauma, pneumonia, and most commonly atelectasis with an underlying etiology such as spontaneous or iatrogenic pneumothorax, massive pleural effusion, or airway obstruction. ${ }^{5}$ In our case, the residual lobes were in the proper position at the end of the right upper lobectomy. Although these lobes hardly remained inflated by postoperative day 2 , the patient became sick. Bronchoscopy and additional drainage could not demonstrate the cause of the lobar atelectasis. The altered vasculature on chest radiography after the tube insertion suggests that the torsion may have occurred while the lobes were being reexpanded by this additional suction. This unusual cause and process made the diagnosis of lobar torsion difficult and delayed.

In the case of postoperative lobar atelectasis, rigorous radiologic and bronchoscopic evaluation to identify the underlying etiology and its appropriate management is crucial. This case also tells us that lobar torsion can occur during the reexpansion of collapsed residual lobes. To avoid this complication, the residual lobe should be fixed, as is widely recommended. ${ }^{1,4}$ In addition, suction should be applied to the chest tube for long enough to keep the lung inflated after long-term compression.

\section{References}

1. Cable DG, Deschamps C, Allen MS, Miller DL, Nichols FC, Trastek VF, et al. Lobar torsion after pulmonary resection: presentation and outcome. J Thorac Cardiovasc Surg. 2001;122:1091-3.

2. Felson B. Lung torsion: radiographic findings in nine cases. Radiology. 1987;162: 631-8.

3. Oddi MA, Taugott RC, Will RJ, Simmons RA, Treasure RL, Schuchmann GF. Unrecognized intraoperative torsion of the lung. Surgery. 1981;89:390-3.

4. Larsson S, Lepore V, Dernevik L, Nilsson F, Selin K. Torsion of a lung lobe: diagnosis and treatment. Thorac Cardiovasc Surg. 1988;36:281-3.

5. Moser ES Jr, Proto AV. Lung torsion: case report and literature review. Radiology. $1987 ; 162: 639-43$.

\title{
Novel sequential bridge to lung transplant in an awake patient
}

\author{
Nikhil P. Patil, MS, MRCS (Eng), MCh (CTVS), ${ }^{\mathrm{a}}$ Aron F. Popov, MD, PhD, ${ }^{\mathrm{a}}$ \\ Nicholas J. Lees, MBChB, FRCA, FFICM, ${ }^{b}$ and André R. Simon, MD, PhD, ${ }^{a}$ London, United Kingdom
}

\footnotetext{
From the Department of Cardiothoracic Transplantation and Mechanical Support, ${ }^{2}$ and the Department of Anaesthesia and Critical Care, ${ }^{\mathrm{b}}$ Royal Brompton and Harefield NHS Foundation Trust, Harefield Hospital, London, UK.

Disclosures: Authors have nothing to disclose with regard to commercial support.

Received for publication July 3, 2014; revisions received Sept 4, 2014; accepted for publication Oct 5, 2014; available ahead of print Nov 7, 2014

Address for reprints: Nikhil P. Patil, MS, MRCS (Eng), MCh (CTVS), Department of Cardiothoracic Transplantation and Mechanical Circulatory Support, Harefield Hospital, London, United Kingdom (E-mail: n.patil@rbht.nhs.uk).

J Thorac Cardiovasc Surg 2015;149:e2-4

$0022-5223 / \$ 36.00$

Copyright (c) 2015 by The American Association for Thoracic Surgery

http://dx.doi.org/10.1016/j.jtcvs.2014.10.031
}

Patients awaiting lung transplant (LTx) may have acute deterioration, but preoperative invasive mechanical ventilation (IMV) is undesirable because it increases mortality associated with LTx. ${ }^{1}$ We describe our experience of bridging to LTx while avoiding IMV by using the Hemolung (ALungTechnologies, Pittsburgh, Pa), a novel extracorporeal carbon dioxide removal $\left(\mathrm{ECCO}_{2} \mathrm{R}\right)$ device for partial respiratory support, with subsequent extracorporeal membrane oxygenation (ECMO) through in situ Hemolung cannulas for escalated support. 


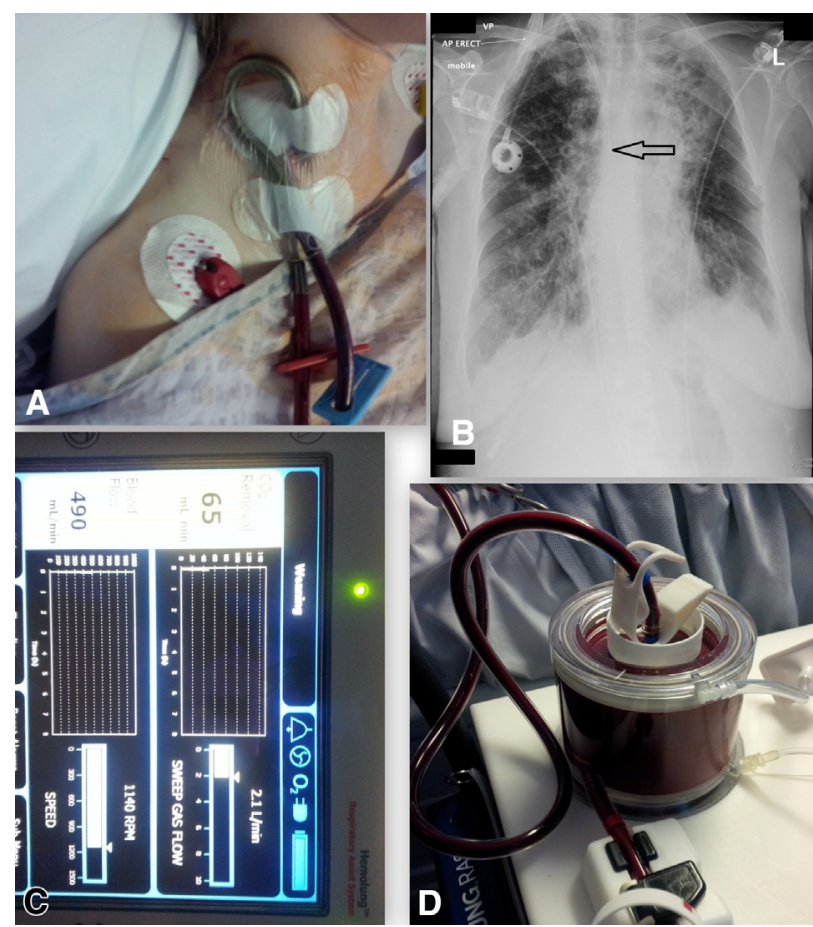

FIGURE 1. A, Hemolung (ALungTechnologies, Pittsburgh, Pa) duallumen cannula inserted through the right internal jugular vein. B, Chest radiograph showing the tip of the Hemolung cannula (arrow) near the superior cavoatrial junction. C, Hemolung controller display. D, Hemolung circuit inflow and outflow cannulas.

\section{CLINICAL SUMMARY}

A 38-year-old woman awaiting LTx was admitted with type 2 respiratory failure following an infectious exacerbation of cystic fibrosis. Despite antibiotics and continuous noninvasive ventilation (NIV) with nasal biphasic positive airway pressure (BiPAP), her condition worsened $\left(\mathrm{PaCO}_{2}\right.$ $9.5 \mathrm{kPa}[71.2 \mathrm{~mm} \mathrm{Hg}]$ and $\mathrm{PaO}_{2} 11 \mathrm{kPa}[82.5 \mathrm{~mm} \mathrm{Hg}]$ on oxygen flow of $6 \mathrm{~L} / \mathrm{min}$ ), and the institutional multidisciplinary team decided to urgently implant the Hemolung as a bridge to LTx.

The Hemolung respiratory assist system combines a centrifugal blood pump (priming volume, $260 \mathrm{~mL}$ ), a gas exchange membrane (surface area, $0.59 \mathrm{~m}^{2}$ ), and a single venovenous (femoral to jugular) dual-lumen $15.5 \mathrm{~F}$ catheter, providing real time measurement of $\mathrm{ECCO}_{2} \mathrm{R}$. With local anesthesia (patient awake and unsedated), the duallumen Hemolung catheter was introduced (heparin bolus of $80 \mathrm{U} / \mathrm{kg}$ intravenously, activated clotting time $>250$ seconds) through the right internal jugular vein (Figure 1, A) and positioned with its tip near the superior cavoatrial junction (Figure 1,B). The proximal and distal (tip) lumens were connected to the Hemolung device inflow and outflow channels, respectively (Figure 1,C). Initial Hemolung blood flow was adjusted to 450 to 500 $\mathrm{mL} / \mathrm{min}$ at $1140 \mathrm{rpm}$, and gas flow (sweep) was adjusted to $2 \mathrm{~L} / \mathrm{min}$, with calculated $\mathrm{ECCO}_{2} \mathrm{R}$ approximately 60 to $70 \mathrm{~mL} / \mathrm{min}$ (Figure 1, D). The $\mathrm{PaCO}_{2}$, previously $9.5 \mathrm{kPa}(71.2 \mathrm{~mm} \mathrm{Hg})$, decreased to $7.95 \mathrm{kPa}(59.6 \mathrm{~mm}$ $\mathrm{Hg})$ with $\mathrm{a} \mathrm{PaO}_{2}$ of $10.8 \mathrm{kPa}(81 \mathrm{~mm} \mathrm{Hg})$ in 1 hour and to $7.45 \mathrm{kPa}(55.8 \mathrm{~mm} \mathrm{Hg})$ with a $\mathrm{PaO}_{2}$ of $9.5 \mathrm{kPa}(71.2$ $\mathrm{mm} \mathrm{Hg}$ ) within 2 hours, and BiPAP NIV could be discontinued. The patient self-ventilated overnight but subsequently became hypercapneic $\left(\mathrm{pH} 7.32, \mathrm{PaCO}_{2} 10 \mathrm{kPa}\right.$ [75 $\mathrm{mm} \mathrm{Hg}$ ], and $\left.\mathrm{PaO}_{2} 13.3 \mathrm{kPa}[99.75 \mathrm{~mm} \mathrm{Hg}]\right)$ the next morning. Hemolung flows were increased, but despite maximal Hemolung flows (gas flow of $10 \mathrm{~L} / \mathrm{min}$, blood flow of $600 \mathrm{~mL} / \mathrm{min}$ at $1300 \mathrm{rpm}$, and calculated $\mathrm{ECCO}_{2} \mathrm{R}$ of approximately 80 to $85 \mathrm{~mL} / \mathrm{min}$ ), the $\mathrm{PaCO}_{2}$ continued to rise to $10.5 \mathrm{kPa}(78.75 \mathrm{~mm} \mathrm{Hg})$ with a $\mathrm{pH}$ of 7.31 and a $\mathrm{PaO}_{2}$ of $13.3 \mathrm{kPa}(99.75 \mathrm{~mm} \mathrm{Hg})$, necessitating resumption of BiPAP NIV. Despite BiPAP and maximum Hemolung therapy, however, the patient continued to be hypercapneic $\left(\mathrm{pH} 7.38, \mathrm{PaO}_{2} 12 \mathrm{kPa}\left[90 \mathrm{~mm} \mathrm{Hg}\right.\right.$ ], $\mathrm{PaCO}_{2} 8.4 \mathrm{kPa}[63 \mathrm{~mm}$ $\mathrm{Hg}]$ ), with impending need for IMV. To avoid IMV if possible, it was decided to escalate therapy to venovenous ECMO (vv-ECMO) with the in situ Hemolung cannulae. The Hemolung circuit was changed to a CentriMagLevitronix (Thoractec Corp, Pleasanton, Calif) vv-ECMO circuit by connecting 3/8-inch inflow and outflow tubing to the existing $15.5 \mathrm{~F}$ dual-lumen cannula. Maximum flow of $1.2 \mathrm{~L} / \mathrm{min}$ was achieved (Figure 2, solid arrows), with eucapnea $\left(\mathrm{PaCO}_{2}\right.$ of $\left.5.0 \mathrm{kPa}[37.5 \mathrm{~mm} \mathrm{Hg}]\right)$. We subsequently aimed for a maintenance $\mathrm{PaCO}_{2}$ of approximately $6 \mathrm{kPa}(45 \mathrm{~mm} \mathrm{Hg})$ to avoid alkalosis. With this strategy, the patient's condition improved, with acceptable, stable blood gas values $\left(\mathrm{pH} 7.33-7.40, \mathrm{PaCO}_{2} 5.0-6.0 \mathrm{kPa}\right.$ [37.5$45 \mathrm{~mm} \mathrm{Hg}$ ], and $\mathrm{PaO}_{2} 11-14 \mathrm{kPa}$ [82.5-105 mm $\left.\mathrm{Hg}\right]$ ), and the BiPAP NIV could be discontinued after 6 hours. The patient's name remained highlighted on the active LTx waiting list while she was supported by vv-ECMO, self-ventilating (off NIV) satisfactorily without evidence of hemolysis (daily plasma free hemoglobin of $<0.1$ $\mathrm{mg} / \mathrm{dL}$ and bilirubin unelevated), and she successfully underwent LTx with ECMO removal 7 days after the initial Hemolung therapy.

\section{DISCUSSION}

IMV is known to affect outcomes adversely in patients awaiting LTx. ${ }^{1}$ Various strategies have been used to bridge patients in critical condition to LTx, including $\mathrm{ECMO},{ }^{2}$ but the optimal approach (central vs peripheral and venoarterial vs venovenous) remains contentious. In addition, the complexity, cost, and associated morbidity of ECMO render it less desirable as a first option. The Hemolung respiratory assist system received CE marking for European use in February 2013. Like ECMO, Hemolung therapy entails anticoagulation and a potential risk of bleeding. Whereas previously published reports on the 


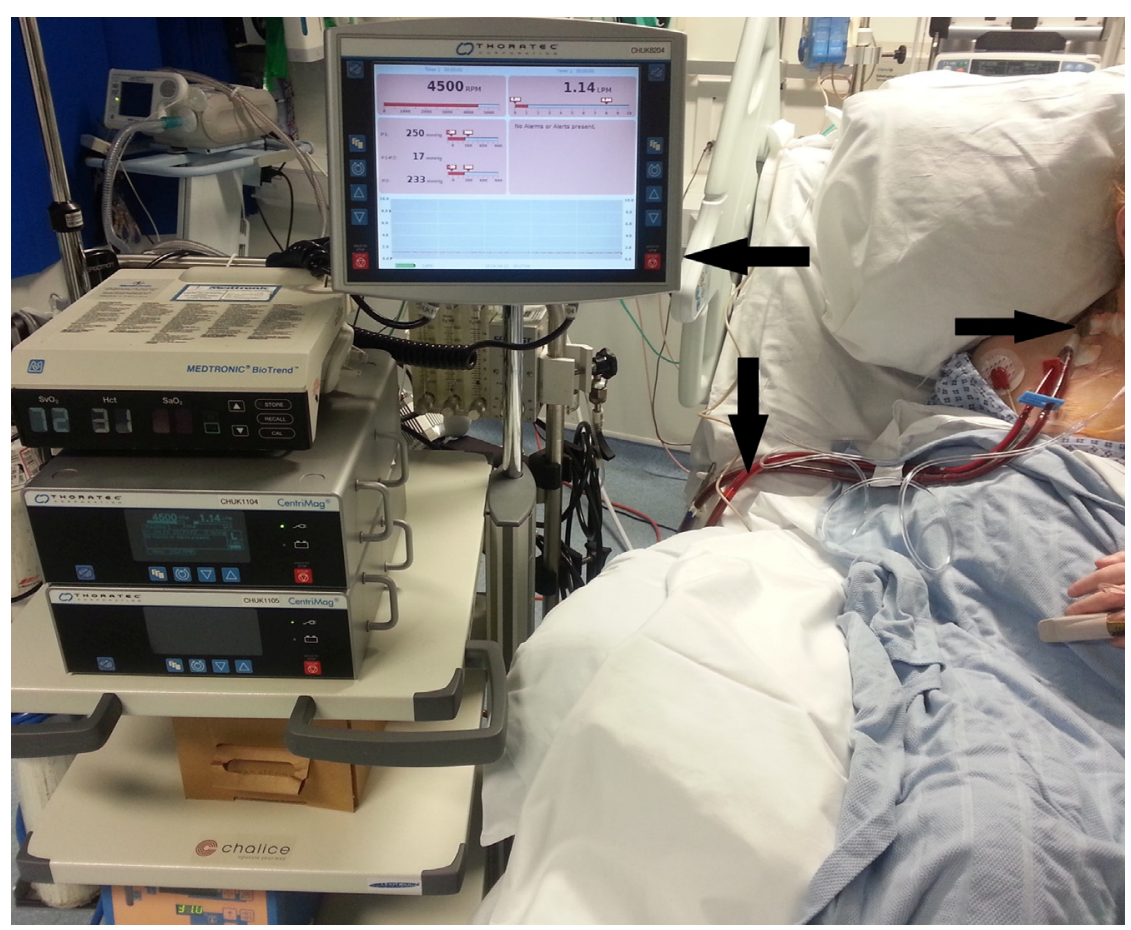

FIGURE 2. In situ Hemolung (ALungTechnologies, Pittsburgh, Pa) cannula used to connect inflow and outflow tubing to venovenous extracorporeal membrane oxygenation circuit (solid arrows).

Hemolung ${ }^{1,3,4}$ elucidated its efficiency in rescuing patients with critical respiratory failure, we found that, although the Hemolung affords ease of access and is good at carbon dioxide removal, it may not be effective enough, because it is limited by the maximum flows that can be achieved. This is the critical "take-home message" to highlight. The Hemolung system has its advantages - easy access, smaller cannula, no rigid need for precise alignment and thus easier insertionhowever, it may ultimately prove insufficient for a patient. We considered exchanging the Hemolung cannula for a larger dual-lumen cannula (eg, Avalon Elite [Avalon Laboratories, Compton, Calif]) but thought that this would be difficult without sedation and intubation and the risk of hemorrhage. We also considered adding a femoral drainage cannula and using the Hemolung cannula as a return but thought that this might hinder mobility and would require new cannulation with inherent risks. We therefore chose the easiest and least invasive option-and it worked. We propose that our strategy of Hemolung therapy for initial rescue in respiratory failure, with escalation if needed to sequential vv-ECMO through the in situ Hemolung dual-lumen cannula, is a clinically safe and effective option for bridging to LTx, as opposed to IMV, which entails different but more serious risks as well as significantly worse patient discomfort and quality of life.

\section{References}

1. Bonin F, Sommerwerck U, Lund LW, Teschler H. Avoidance of intubation during acute exacerbation of chronic obstructive pulmonary disease for a lung transplant candidate using extracorporeal carbon dioxide removal with the Hemolung. J Thorac Cardiovasc Surg. 2013;145:e43-4.

2. Fuehner T, Kuehn C, Hadem J, Wiesner O, Gottlieb J, Tudorache I, et al. Extracorporeal membrane oxygenation in awake patients as bridge to lung transplantation. Am J Respir Crit Care Med. 2012;185:763-8.

3. Lund LW, Federspiel WJ. Removing extra $\mathrm{CO}_{2}$ in COPD patients. Curr Respir Care Rep. 2013;2:131-8.

4. Burki NK, Mani RK, Herth FJ, Schmidt W, Teschler H, Bonin F, et al. A novel extracorporeal $\mathrm{CO}_{2}$ removal system: results of a pilot study of hypercapnic respiratory failure in patients with COPD. Chest. 2013;143:678-86. 